\title{
INVERSE $*$-SEMIGROUPS $*$-GENERATED BY FAMILIES OF ISOMETRIES
}

\author{
WAC $Ł A W ~ S Z Y M A N S K I$
}

(Communicated by John B. Conway)

\begin{abstract}
It is shown that if a *-semigroup *-generated by a family of commuting Hilbert space isometries that commute each other, none of which commutes with the adjoint of another one, and none of which is a nonzero power of another one, consists of partial isometries, then it is singly *-generated. Also, the following result on algebraic semigroups is proved: If $S$ is an inverse *semigroup *-generated by a set $X$ satisfying the generating relations: $a^{*} a=1$, $a b=b a$, for all $a, b \in X$, then $S$ is the bicyclic semigroup. Both results follow from the special behavior of inverse *-semigroups *-generated by analytic Toeplitz operators.
\end{abstract}

\section{INTRODUCTION}

In this paper all semigroups have unit. A semigroup $S$ is a $*$-semigroup if there is a mapping $*: S \rightarrow S$ satisfying $(a b)^{*}=b^{*} a^{*},\left(a^{*}\right)^{*}=a$ for $a, b \in S$, $1^{*}=1$. Let $X$ be a subset of a *-semigroup $S . S^{*}(X)$ denotes the *semigroup that consists of all words in $a, b^{*}(a, b \in X)$. It will be understood that $1 \in S^{*}(X)$, and thus 1 will not be listed as an element of $X$. Elements of $X$ may be related to each other by certain equalities between words in $a, b^{*}(a, b \in$ $X)$. Such relations are called generating relations for the semigroup $S^{*}(X)$ (cf. [1], p. 41). $S$ is *-generated by $X$ if $S=S^{*}(X)$. If $X=\left\{a_{1}, \ldots, a_{k}\right\}$, then $S^{*}\left(a_{1}, \ldots, a_{k}\right)=S^{*}(X)$. The number of letters in a word will refer only to the letters, not to their powers, e.g. $a b^{2} a^{* 3}$ is a 3-letter word. Let $S$ be a semigroup. An element $b \in S$ is called the (semigroup) inverse of $a \in S$ if $a b a=a, b a b=b . S$ is called an inverse semigroup if each element of $S$ has a unique inverse.

1.1. Fundamental characterization of inverse semigroups ([1], Theorem 1.17). $A$ semigroup $S$ is an inverse semigroup if and only if every element of $S$ has an inverse and all idempotents in $S$ commute.

Let $H$ be a complex Hilbert space. $B(H)$ stands for the *-semigroup of all linear bounded operators in $H$ with the composition and with $*$ being the

Received by the editors January 27, 1989.

1980 Mathernatics Subject Classification (1985 Revision). Primary 47D05, 20M05, 47B35, $20 \mathrm{M} 18$. 
adjoint operation. *-subsemigroups of $B(H)$ will be allowed to contain all appropriate scalar multiples of their members. $U \in B(H)$ is a partial isometry if $U U^{*} U=U$, i.e. if $U^{*}$ is a semigroup inverse of $U$. Each inverse semigroup is isomorphic with a $*$-semigroup of partial isometries in a Hilbert space. Also, each *-semigroup of partial isometries in a Hilbert space is an inverse semigroup (cf. [4] Proposition 1.1 for the proof and references). Partial isometries $U \in$ $B(H)$ for which $S^{*}(U)$ is an inverse semigroup are completely characterized in [2].

The bicyclic semigroup is one of the fundamental examples of an inverse semigroup. It is defined as the free semigroup $S^{*}(a)$ *-generated by a single element $a$ such that $a^{*} a=1$ (cf. [1], p. 43). Consider a more general question: Suppose $S=S^{*}(X)$ is a $*$-semigroup and $X$ satisfies the generating relations $a^{*} a=1, a b=b a$, for all $a, b \in X$. What are the consequences? The answer is given in $\S 3$. It turns out that $S^{*}(X)$ is *-generated by a single element, hence it is the bicyclic semigroup. In $\S 2$ inverse $*$-semigroups generated by analytic Toeplitz operators are studied.

$C$ denotes the complex plane, $N$ stands for the set of all non-negative integers. An operator $P \in B(H)$ is a projection if $P=P^{2}=P^{*}$.

\section{INVERSE *-SEMIGROUPS GENERATED BY ANALYTIC TOEPLITZ OPERATORS}

The results of this section concern the classical Hardy space on the unit disc. The notations and terminology are standard (cf., e.g. [5]). $\Gamma$ is the unit circle, $m$ is the normalized Lebesgue measure on $\Gamma, z$ denotes the identity function on $\Gamma, P$ is the Riesz projection $=$ the projection of $L^{2}$ onto $H^{2}$. A function $q \in H^{2}$ is inner if $|q|=1, m$ - a.e. If $q \in H^{\infty}$, then $T_{q}: H^{2} \rightarrow H^{2}$ denotes the analytic Toeplitz operator $T_{q} f=q f, f \in H^{2}$. The following theorem is the main result of this section:

2.1. Theorem. Suppose $X$ is a family of isometries in $H^{2}$ that commute with the unilateral shift $T_{z}$. If the *-semigroup $S^{*}\left(X \cup\left\{T_{z}\right\}\right)$ *-generated by $T_{z}$ and all isometries from $X$ is an inverse semigroup, then $S^{*}\left(X \cup\left\{T_{z}\right\}\right)=S^{*}\left(T_{z}\right)$, i.e. is *-generated by $T_{z}$ alone.

It will be convenient to prepare the proof by proving separately the following lemma, perhaps of interest on its own.

2.2. Lemma. Let $q$ be an inner function. Let $k \in N$. The projections $T_{z}^{k} T_{z}^{k *}$, $T_{q} T_{q}^{*}$ commute if and only if $q \in z^{k} H^{2}$ or $q=c z^{p}$ with some $c \in \Gamma, p \in N$, $p<k$.

Proof. Firstly notice that an inner function $q$ is an analytic polynomial if and only if $q=c z^{p}$ with some $c \in \Gamma, p \in N$. This can be shown in an elementary way using the maximum modulus theorem.

Let $q$ now be an inner function. Then $T_{q}$ is an isometry in $H^{2}$. The operators $T_{z}^{k} T_{z}^{k *} f=z^{k} P \bar{z}^{k} f, T_{q} T_{q}^{*} f=q P \bar{q} f, f \in H^{2}$, are projections onto 
$\operatorname{ran} T_{z}^{k}=z^{k} H^{2}$ and $\operatorname{ran} T_{q}=q H^{2}$, respectively. Let $q=q_{0}+q_{1} z+\cdots$ be the Fourier expansion of $q$. For the sake of convenience denote:

$$
L=T_{z}^{k} T_{z}^{k *} T_{q} T_{q}^{*}, \quad R=T_{q} T_{q}^{*} T_{z}^{k} T_{z}^{k *}
$$

Fix arbitrarily $n \in N$. Then

$$
R z^{n}=0 \quad \text { if } n<k, \quad R z^{n}=q P \bar{q} z^{n} \quad \text { if } n \geq k .
$$

Assume that the projections $T_{z}^{k} T_{z}^{k *}, T_{q} T_{q}^{*}$ commute, i.e. $L=R$. In particular, for the constant function $f=1$ :

$$
0=R 1=L 1=z^{k} P \bar{z}^{k} q P \bar{q} 1=\bar{q}_{0} z^{k} P \bar{z}^{k} q .
$$

Hence, $q_{0}=0$ or $q$ is an analytic polynomial of degree at most $k-1$. By the remark opening the proof, $q_{0}=0$ or $q=c z^{p}$ with some $c \in \Gamma, p \in N$, $p<k$. Suppose $q$ is not an analytic polynomial. Hence $q_{0}=0$. If $k=1$, the proof is finished, because then $q \in z H^{2}$. If $k>1$, take $f=z$. Then $z \bar{q}=\bar{q}_{1}+\bar{q}_{2} \bar{z}+\cdots$ and by $(2.3)$

$$
0=R z=L z=z^{k} P \bar{z}^{k} q P \bar{q} z=\bar{q}_{1} z^{k} P \bar{z}^{k} q \text {. }
$$

Hence, $q_{1}=0$. Thus $q \in z^{2} H^{2}$. If $k=2$, the proof is finished. If not, continue the above process until $k$.

For the proof of the converse assume that $q \in z^{k} H^{2}$ or $q=z^{p}$ with some $p \in N, p<k$. Then $q H^{2} \subset z^{k} H^{2}$ or $z^{k} H^{2} \subset q H^{2}$, respectively. Hence the projections onto these subspaces commute. Q.E.D.

Proof of Theorem 2.1. Every operator in $H^{2}$ commuting with $T_{z}$ is of the form $T_{q}$ with some $q \in H^{\infty}$ ([5], Problem 147). Hence all members of $X$ are of that form. Take arbitrarily $T_{q} \in X$. Since $T_{q}$ is an isometry, $q$ is an inner function. By the fundamental characterization of inverse semigroups (1.1), all idempotents in $S^{*}\left(X \cup\left\{T_{z}\right\}\right)$ commute. In particular, $T_{q} T_{q}^{*}$ commutes with $T_{z}^{k} T_{z}^{k *}$ for each $k \in N$. By Lemma 2.2, $q \in z^{k} H^{2}$ or $q=c z^{p}$ with some $c \in \Gamma, p \in N, p<k$, for each $k \in N$. Since $q \neq 0, q$ cannot belong to all $z^{k} H^{2}, k \in N$. Hence there are $c \in \Gamma, p \in N$ such that $q=c z^{p}$. Q.E.D.

\section{CONSEQUENCES FOR OPERATOR AND ABSTRACT SEMIGROUPS}

The product of Hilbert space isometries is an isometry. Here is a corresponding result for partial isometries.

3.1. Proposition. Suppose $U, V \in B(H)$ are partial isometries. $U V$ is a partial isometry if and only if $U^{*} U$ commutes with $V V^{*}$.

Proof. If $U$ is a partial isometry, then so is $U^{*}$; hence both $E=U^{*} U, F=$ $V V^{*}$ are projections. Suppose $E F=F E$. Then $U V(U V)^{*} U V=U F E V=$ $U E F V=U V$. Hence, $U V$ is a partial isometry. Now suppose that $U V$ is a partial isometry. Then $U F E V=U V$. Multiplying by $U^{*}$ from the left and by 
$V^{*}$ from the right one gets $E F E F=E F$. Thus $E F$ is an idempotent of norm at most one. Such idempotents are self-adjoint. Hence, $E F=F E$. Q.E.D.

The proof of the "only if" part is purely algebraic. The proof of the "if" part is geometric. Is it possible to give an algebraic proof?

3.2. Proposition. Suppose $V, W \in B(H)$ are isometries. If $V W^{*}=W^{*} V$ then $V W=W V, V V^{*}$ commutes with $W$, and $W W^{*}$ commutes with $V$. Conversely, if $V W=W V$, and $V V^{*}$ commutes with $W$ or $W W^{*}$ commutes with $V$, then $V W^{*}=W^{*} V$.

Proof. Suppose $W^{*}$ commutes with $V$. It is proved in [3], Proposition 3.5, that $W$ commutes with $V$. Here is that argument, for the sake of completeness: $(V W-W V)^{*}(V W-W V)=W^{*} V^{*} V W-W^{*} V^{*} W V-V^{*} W^{*} V W+V^{*} W^{*} W V$ $=0$. From this it is clear that $V V^{*}$ commutes with $W$, and $W W^{*}$ commutes with $V$. For the converse compute $\left(V^{*} W-W V^{*}\right)^{*}\left(V^{*} W-W V^{*}\right)=$ $W^{*} V V^{*} W-W^{*} V W V^{*}-V W^{*} V^{*} W+V W^{*} W V^{*}=0$ if $V V^{*}$ commutes with $W$. If $W W^{*}$ commutes with $V$, then replace $W$ by $V$ and $V$ by $W$ in the above computation. Q.E.D.

The above proof uses not only the *-semigroup structure of $B(H)$, but also the $C^{*}$-algebra structure of $B(H)$. Can this property be proved in a purely *-semigroup context?

3.3. Proposition. Let $V, W \in B(H)$ be isometries such that $V W^{*}=W^{*} V$. Then the semigroup $S^{*}(V, W)$ consists of partial isometries, i.e. is an inverse semigroup.

Proof. By Proposition 3.2, $V W=W V$. hence, all members of $S^{*}(V, W)$ have form $U=V^{j} V^{* k} W^{m} W^{* n}, j, k, m, n \in N$. It is immediate that $U U^{*} U=U$. Now $S^{*}(V, W)$ is an inverse semigroup, because it is a *-semigroup consisting of partial isometries-use a result of Duncan-Paterson quoted in $\S 1$. Q.E.D.

Now fix two isometries $V, W \in B(H)$ such that $V$ commutes with $W$, but not with $W^{*}$. All words in $V, V^{*}$ have form $V^{k} V^{* j}, j, k \in N$, all words in $W, W^{*}$ have form $W^{m} W^{* n}, m, n \in N$. Hence, all elements of the *semigroup $S^{*}(V, W)$ are finite products of the form $V^{k} V^{* j} W^{m} W^{* n} \ldots$. If two words in $S^{*}(V, W)$ are equal, then this equality can always be reduced to an equality between two words in, at most, two letters each. This is done as follows: suppose $w, w^{\prime}$ are words in $S^{*}(V, W)$ of at least two letters each, and $w=w^{\prime}$. Multiplying both sides of $w=w^{\prime}$ by a suitable power of $V, V^{*}, W$, or $W^{*}$, and using the relations $V^{*} V=I, W^{*} W=I, V W=W V$, one gets new words $v, v^{\prime}$ such that $v=v^{\prime}$, at least one of $v, v^{\prime}$, say $v$, has the number of letters strictly less than $w$, and the number of letters in $v^{\prime}$ does not exceed the number of letters in $w^{\prime}$. Therefore all generating relations for the semigroup $S^{*}(V, W)$ must be expressed as equalities between at most two-letter words. The above procedure can be proved by considering cases of possible endings (or beginnings) of words. Instead of this not very interesting 
proof, here is a demonstration of that "shortening of words" effect. Suppose $W^{* p} V^{k} V^{* j}=V^{* m} W^{n} W^{* q} V^{s}, j, k, m, n, p, s \in N$. Multiply from the right by $V^{j} W^{q}$. Then $W^{* p} W^{q} V^{k}=V^{* m} V^{s+j} W^{m}$. The words on both sides are now two-letter words in the letters depending on powers. This effect is purely algebraic and depends only on the above three relations.

3.4. Lemma. Suppose $V, W \in B(H)$ are isometries, $V W=W V, V^{*} W \neq$ $W V^{*}$, and no nonzero power of $W$ equals a power of $V$. Then the relations:

$$
V^{*} V=I, \quad W^{*} W=I, \quad V W=W V
$$

are the only generating relations for the *-semigroup $S^{*}(V, W)$.

Proof. Notice that $W$ is not unitary. If $W$ was unitary, then Fuglede's theorem would imply $V^{*} W=W V^{*}$. By the remarks before this theorem it is enough to consider equalities between words of at most two letters each. It is not difficult to show that either they contradict some assumptions about $V, W$, or they imply one of the conditions $(\mathrm{G})$. Any equality between one-letter words leads to trivialities or contradictions, e.g. $V^{* k}=W^{j}$ cannot happen, because the ranges of $V^{* k}$ and $W^{j}$ are different, unless $k=j=0$. A similar conclusion is true if one examines equalities between a one-letter word and a two-letter word. The only nontrivial equality between two-letter words, which is not already in $(G)$, is $V^{* k} W^{n}=W^{n} V^{* k}, k, n \in N$. Assume $k, n \geq 1$. Multiply this equality by $V^{k-1}$ from the right and by $W^{* n-1}$ from the left. then $V^{*} W=W V^{*}$, which contradicts the assumption. Q.E.D.

3.5. Theorem. Suppose $X$ is a family of commuting isometries on a Hilbert space $H$. Assume that

(a) no element of $X$ commutes with the adjoint of another element of $X$, and

(b) for any $V, W \in X$, no nonzero power of $V$ equals a power of $W$. If $S^{*}(X)$ consists of partial isometries (is an inverse semigroup), then $X$ has one element.

Proof. Suppose there are two different $V, W \in X$. By Lemma 3.4, the only generating relations for $S^{*}(V, W)$ are the relations $(\mathrm{G})$. Choose an inner function $q$ which is not a polynomial. Then $T_{z}$ and $T_{q}$ satisfy the assumption of Lemma 3.4. Hence, the generating relations for the *-semigroup $S^{*}\left(T_{z}, T_{q}\right)$ are exactly the same as for $S^{*}(V, W)$. Thus the mapping $h(V)=T_{z}, h(W)=T_{q}$ extends to a *-preserving isomorphism of $S^{*}(V, W)$ and $S^{*}\left(T_{z}, T_{q}\right)$. Since $S^{*}(V, W)$ consists of partial isometries, it follows from Proposition 3.1 that $W W^{*}$ commutes with $V^{k} V^{* k}$ for each $k \in N$, because $W^{*} V^{k}$ is a partial isometry. Hence, $T_{q} T_{q}^{*}$ commutes with $T_{z}^{k} T_{z}^{k *}$ for each $k \in N$. It follows from the proof of Theorem 2.1 that $q=c z^{p}$ with some $c \in \Gamma, p \in N$, which is a contradiction. Q.E.D.

In a very similar manner one proves the following general result for algebraic semigroups. 
3.6. Theorem. If $S$ is an inverse semigroup *-generated by a set $X$ which satisfies the generating relations: $a^{*} a=1, a b=b a$, for $a, b \in X$, then $X$ has one element and $S$ is the bicyclic semigroup.

Proof. As above, suppose $X$ has two different elements $a, b$. Let $q$ be an inner function chosen as in the proof of Theorem 3.5. Then the *-semigroups $S^{*}(a, b)$ and $S^{*}\left(T_{z}, T_{q}\right)$ are *-isomorphic, because their generating relations are the same, by Lemma 3.4. Here $a$ corresponds to $T_{z}, b$ corresponds to $T_{q}$. By 1.1, the idempotents $b b^{*}, a^{k} a^{* k}$ commute for each $k \in N$, and they belong to $S^{*}(a, b)$. Thus the projections $T_{q} T_{q}^{*}, T_{z}^{k} T_{z}^{* k}$ commute for all $k \in N$. Hence, $q=c z^{p}$ for some $c \in \Gamma, p \in N$, which is a contradiction. Thus $X$ has one element, $X=\{a\}$. Hence, $S=S^{*}(a)$ is bicyclic. Q.E.D.

\section{REFERENCES}

1. A. H. Clifford and G. B. Preston, The algebraic theory of semigroups, Math. Surveys Monographs, Vol. I, no. 7, Amer. Math. Soc., 1961.

2. J. B. Conway, J. Duncan and A. L. T. Paterson, Monogenic inverse semigroups and their C*-algebras, Proc. Roy. Soc. Edinburgh Sect. A 98 (1984), 13-24.

3. J. B. Conway and W. Szymanski, Linear combinations of hyponormal operators, Rocky Mountain J. Math., 18 (1988), 695-705.

4. J. Duncan and A. L. T. Paterson, C*-algebras of inverse semigroups, Proc. Edinburgh Math. Soc. 28 (1985), 41-58.

5. P. R. Halmos, $A$ Hilbert space problem book (2nd ed.), Springer Verlag, New York, Heidelberg, Berlin, 1982, GTM 19.

Department of Mathematics and Computer Science, West Chester University, West Chester, Pennsylvania 19383 